\title{
The Research of Tight MINS/GPS Integrated navigation System Based Upon Date Fusion
}

\author{
Hao YAN ${ }^{1, a}$, Kai LIU ${ }^{1, b}$ and Hua CHE ${ }^{1, c}$ \\ ${ }^{1}$ The First Aeronautic Institution of Air Force, Department of instrument and electrical equipment, \\ Flight control office, HeNan, XinYang 464000

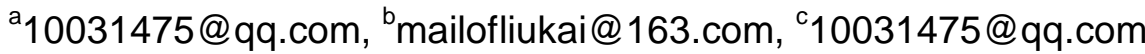

Keywords: MINS/GPS; integrated mode; filter algorithm; pseudo range difference.

Abstract. In this paper, loose, tight and super tight integrated MINS/GPS mode's structure and working principle have been discussed. The Kalman filter module is set up based upon the data fusion theory. The characteristic of tight integrated MINS/GPS system's data fusion has been researched through hardware-in-the-loop experiment.

\section{Introduction}

With the development of modern navigation system, the integrated navigation system has become the most popular mode. Micro inertial strap down navigation (MINS) and global position system (GPS) form the integrated navigation system. Its cost is low, capacity is small, weight is light, power is low, and has excellent compound performance advantage. Nowadays, it is widely used in the fields of automobile, robot, armament and UAV.

MINS based upon MIMU design has inherited the advantage of strap down inertial system such as good independence and anti-disturbance. On the other hand, the precision of micro inertial sensor is not high, the system error will accumulate with time goes on. It is difficult to finish long-time measure and navigation mission and need external information fusion. GPS has the advantage such as high precision and stable error, but at the same time it also has some shortcoming such as poor independence and anti-disturbance. In the MINS/GPS integrated system, through measuring pseudo range and pseudo range ratio to form tight integrated mode, it can form one kind of compound mode that has high precision and reliability. In this paper, based upon present mature loose integrated theory, the tight mode can be researched and simulated through hardware-in-the-loop experiment.

\section{MINS/GPS integrated navigation principle}

In the GPS and inertial navigation system, depending upon the extend of information exchange, it can be classified as three forms: loose, tight and super tight integrated. The loose system based upon location and velocity is one kind of integrated method that can be fulfilled more easily. Inertial system and GPS are working independently. When the GPS is working, the fusion algorithm will make the output of inertial navigation system adjust to be consistent with the GPS. At the same time, it will feedback the inertial system with optimal estimate. The system's working principle is illustrated as Fig.1.

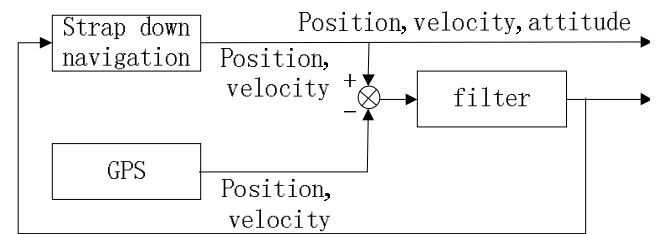

Fig.1 loose integrated MINS/GPS block diagram

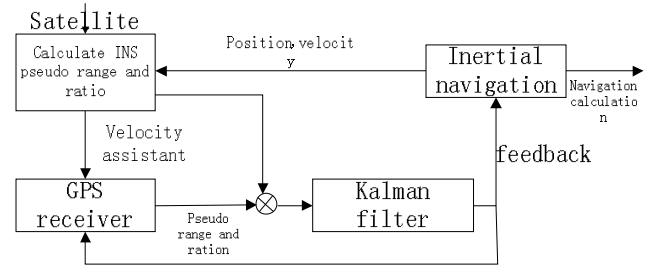

Fig.2 tight integrated MINS/GPS block diagram

The tight integrated system based upon pseudo range and ration mainly utilizes the original pseudo range and ration information from GPS receiver. The GPS receiver assists INS with each other. GPS receiver provides pseudo range carrier phase and Doppler frequency shift information. These signal 
will send to one filter. The error signal includes inertial system and GPS receiver error. The Kalman filter will provide the best navigation result. System working principle is shown as Fig.2.

Super tight system have the advantage of loose and tight system at the same time. In its tracking loop, directly utilize MIMU value to measure assistant GPS code tracking loop and carrier tracking loop. One integrated Kalman filter feedback is used to take place of traditional tracking loop. System's working principle is shown as Fig.3.

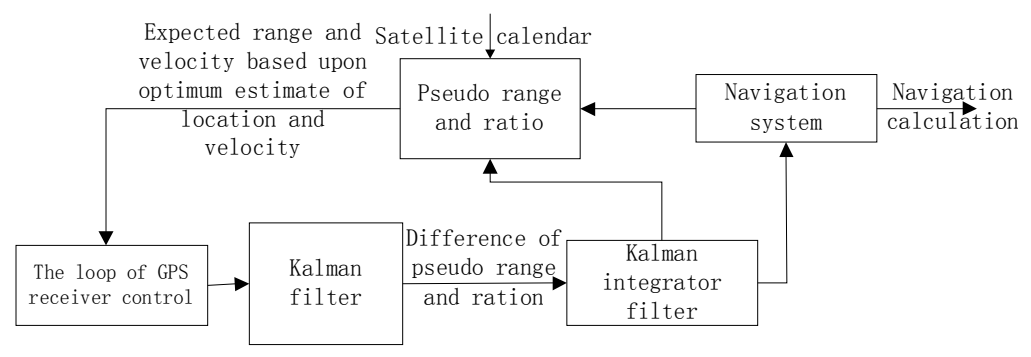

Fig.3 super tight integrated MINS/GPS block diagram

From above structure, the loose integrated system has simple structure and less amount of calculation. In application, it is more easily to be realized. It can satisfied the system that has more demand on real time feature. Nowadays, it has already applied widely. Super tight system although has overwhelming advantages, but it will refer to the internal arrangement of GPS receiver. It is more difficult to be realized. Limited on the research ability on receiver, the research on super tight MINS/GPS is still in the simulation period. There is still a long distance with the real engineering application. The tight integrated system has no connecting with the arrangement of GPS receiver. So when the visual satellites become less, it still can keep some kind of precision in a short time. The system has excellent precision and stability.

\section{Date fusion of MINS/GPS integrated navigation system}

When tackling with the data of GPS and inertial system, the all sensor information that can be got should be considered. In this assumption, the optimum estimate can be achieved about the location, velocity and attitude. The most common used tool is the Kalman filter that is used to fuse dynamic redundancy multi sensors data in real time. Based upon the assumption that the measurement model is already known, the static optimum estimate can be resolved. When the visual satellites is more than 4 in GPS system, the Kalman filter about location and velocity in loose system has already used in many mature systems. In this paper, the satellites selecting algorithm and pseudo range ration state equation of tight system are discussed. In the tight system, the main problems are located in the data tackling method. In the optimum combination of system, it will encounter the so called "dimensional disaster". When researching about the combination data tackling, the scattered filter algorithm should be paid more attention. Through the simulation of different algorithm of data fusion, the optimum data fusion can be found.

\section{state equation about tight integrated system of pseudo range and ration MINS/GPS}

The error state of MINS include velocity error, attitude error, location error, gyroscope drift and accelerometer zero-offset. Making the reference of loose system state matrix adopting principle, the GPS error state, tight system state matrix $\mathrm{F}$ has added pseudo range and pseudo range ration's error variable. It mainly consider about two errors concerning about time. One is the equivalent range error $\delta$ tu caused by clock time, the other is the velocity error $\delta$ tru caused by clock frequency. $\delta$ tru is the first order Markov process.

Take the inertial navigation system's error state value and GPS system's error value as the integrated system's state, union the MINS error state equation and GPS error state equation, the integrated system state equation can be obtained. 


$$
\left[\begin{array}{l}
X_{I}^{\mathcal{Q}_{I}}(t) \\
X_{G}^{\mathcal{Q}_{G}}(t)
\end{array}\right]=\left[\begin{array}{cc}
F_{I}(t) & 0 \\
0 & F_{G}(t)
\end{array}\right]\left[\begin{array}{l}
X_{I}(t) \\
X_{G}(t)
\end{array}\right]+\left[\begin{array}{cc}
G_{I}(t) & 0 \\
0 & G_{G}(t)
\end{array}\right]\left[\begin{array}{l}
W_{I}(t) \\
W_{G}(t)
\end{array}\right]
$$

And,

$$
\begin{aligned}
& X=\left[\begin{array}{llllllllllllllllllll}
\varphi_{E} & \varphi_{N} & \varphi_{U} & \delta v_{E} & \delta v_{N} & \delta v_{U} & \delta L & \delta \lambda & \delta h & \varepsilon_{b x} & \varepsilon_{b y} & \varepsilon_{b z} & \varepsilon_{r x} & \varepsilon_{r y} & \varepsilon_{r z} & \nabla_{x} & \nabla_{y} & \nabla_{z} & \delta t_{u} & \delta t_{r u}
\end{array}\right]^{T} \\
& F_{I}=\left[\begin{array}{cc}
F_{N} & F_{s} \\
0 & F_{M}
\end{array}\right]_{18 \times 18}, F_{s}=\left[\begin{array}{ccc}
C_{b}^{n} & C_{b}^{n} & 0_{3 \times 3} \\
0_{3 \times 3} & 0_{3 \times 3} & C_{b}^{n} \\
0_{3 \times 3} & 0_{3 \times 3} & 0_{3 \times 3}
\end{array}\right]_{9 \times 9}, \quad G_{I}=\left[\begin{array}{ccc}
C_{b}^{n} & 0_{3 \times 3} & 0_{3 \times 3} \\
0_{9 \times 3} & 0_{9 \times 3} & 0_{9 \times 3} \\
0_{3 \times 3} & I_{3 \times 3} & 0_{3 \times 3} \\
0_{3 \times 3} & 0_{3 \times 3} & I_{3 \times 3}
\end{array}\right]_{18 \times 9}, \text {, } \\
& X_{G}(t)=\left[\begin{array}{ll}
\delta t_{u} & \delta t_{r u}
\end{array}\right]^{T}, F_{G}(t)=\left[\begin{array}{cc}
1 & 0 \\
0 & -\beta_{t r u}
\end{array}\right], G_{G}(t)=\left[\begin{array}{ll}
1 & 0 \\
0 & 1
\end{array}\right], W_{G}(t)=\left[\begin{array}{cc}
\omega_{t u} & -\omega_{t r u}
\end{array}\right]^{T}
\end{aligned}
$$

$F_{N}$ is the error matrix of $9 \times 9$ dimension inertial navigation system. It is corresponding with 9 basic navigation parameters matrix. Inertial navigation system's white noise is taken from the gyro's three axis's random white noise, first order Markov driven white noise and accelerometer random white noise, that is

$$
W_{I}(t)=\left[\begin{array}{lllllllll}
\omega_{g x} & \omega_{g y} & \omega_{g z} & \omega_{r x} & \omega_{r y} & \omega_{r z} & \omega_{a x} & \omega_{a y} & \omega_{a z}
\end{array}\right]_{9 \times 1}^{T}
$$

\section{pseudo range/pseudo range ration integrated MINS/GPS tight system measurement equation}

In the tight integrated system based upon pseudo range and pseudo range ration, the pseudo range value is the pseudo information calculated from the output of MINS navigation parameters, then the minus between this value and pseudo range of GPS receiver can be obtained. The pseudo range ration is also the difference between inertial system and GPS output. In the following content, the system measurement equation can be derived from the two respects.

When MINS/GPS tight system in on operation, GPS receiver will select the best 4 or more satellites through $\mathrm{G}$ matrix satellite selecting algorithm to calculate carrier's location and clock error, here $\mathrm{i}=1,2,3,4$.

The observing equation of system pseudo range is as following:

$$
Z_{\rho}(t)=H_{\rho}(t) X(t)+V_{\rho}(t)
$$

$$
\begin{aligned}
& \text { And, } H_{\rho}(t)=\left[\begin{array}{llll}
0_{4 \times 6} & H_{\rho 1} & 0_{4 \times 6} & H_{\rho 2}
\end{array}\right], H_{\rho 1}=\left[\begin{array}{ccc}
a_{11} & a_{12} & a_{13} \\
& \mathrm{M} & \\
a_{41} & a_{41} & a_{41}
\end{array}\right]_{4 \times 3}, H_{\rho 2}=\left[\begin{array}{cc}
1 & 0 \\
\mathrm{M} & \mathrm{M} \\
1 & 0
\end{array}\right]_{4 \times 2} \text {, } \\
& \left\{\begin{array}{c}
a_{i 1}=\left(R_{N}+h\right)\left[-e_{i 1} \sin L \cos \lambda-\sin L \sin \lambda\right]+\left[R_{N}\left(1-e^{2}\right)+h\right] e_{i 3} \cos L \\
a_{i 2}=\left(R_{N}+h\right)\left[e_{i 2} \cos L \cos \lambda-\cos L \sin \lambda\right] \\
a_{i 3}=e_{i 1} \cos L \cos \lambda+e_{i 2} \cos L \sin \lambda
\end{array}, i=1,2,3,4\right.
\end{aligned}
$$

In the equation, $e_{i 1} 、 e_{i 2} 、 e_{i 3}$ is the cosine value between the i satellite and client.

Be similar with the pseudo range observing value, the observing equation of system pseudo range ration is

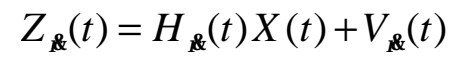

$$
\begin{aligned}
& \text { and, } H_{\alpha \propto}(t)=\left[\begin{array}{llll}
0_{4 \times 3} & H_{\alpha 4} & 0_{4 \times 9} & H_{\alpha Q 2}
\end{array}\right], H_{\alpha Q}=\left[\begin{array}{ccc}
b_{11} & b_{12} & b_{13} \\
& \mathrm{M} & \\
b_{41} & b_{41} & b_{41}
\end{array}\right]_{4 \times 3}, H_{\mathrm{\beta QQ}}=\left[\begin{array}{cc}
0 & 1 \\
\mathrm{M} & \mathrm{M} \\
0 & 1
\end{array}\right]_{4 \times 2} \\
& \left\{\begin{array}{c}
b_{i 1}=-e_{i 1} \sin \lambda+e_{i 2} \cos \lambda \\
b_{i 2}=e_{i 1} \sin L \cos \lambda-e_{i 2} \sin L \sin \lambda+e_{i 3} \cos L, i=1,2,3,4 . \\
b_{i 3}=e_{i 1} \cos L \cos \lambda+e_{i 2} \cos L \sin \lambda+e_{i 3} \sin L
\end{array}\right.
\end{aligned}
$$

So the measurement equation of MINS/GPS tight integrated system can be derived

$$
Z(t)=H(t) X(t)+V(t)
$$

And the observing value $\mathrm{Z}$ is 


$$
\begin{aligned}
& Z=\left[\begin{array}{llllll}
\rho_{I 1}-\rho_{G 1} & \mathrm{~L} & \rho_{I 4}-\rho_{G 4} & \rho_{\gamma_{1}}-\rho_{G 1} & \mathrm{~L} & \rho \alpha_{4}-\rho \sigma_{4}
\end{array}\right]^{T} \\
& H=\left[\begin{array}{l}
H_{\rho} \\
H_{\alpha \times}
\end{array}\right]=\left[\begin{array}{cccccccccccc}
0 & 0 & 0 & 0 & 0 & 0 & a_{11} & a_{12} & a_{13} & \mathrm{~L} & 1 & 0 \\
\mathrm{~L} & \mathrm{~L} & \mathrm{~L} & \mathrm{~L} & \mathrm{~L} & \mathrm{~L} & \mathrm{~L} & \mathrm{~L} & \mathrm{~L} & \mathrm{~L} & \mathrm{~L} & \mathrm{~L} \\
0 & 0 & 0 & 0 & 0 & 0 & a_{41} & a_{42} & a_{43} & \mathrm{~L} & 1 & 0 \\
0 & 0 & 0 & b_{11} & b_{12} & b_{13} & 0 & 0 & 0 & \mathrm{~L} & 0 & 1 \\
\mathrm{~L} & \mathrm{~L} & \mathrm{~L} & \mathrm{~L} & \mathrm{~L} & \mathrm{~L} & \mathrm{~L} & \mathrm{~L} & \mathrm{~L} & \mathrm{~L} & \mathrm{~L} & \mathrm{~L} \\
0 & 0 & 0 & b_{41} & b_{42} & b_{43} & 0 & 0 & 0 & \mathrm{~L} & 0 & 1
\end{array}\right]
\end{aligned}
$$

\section{Simulation test and result analysis}

In this paper, the effectiveness of the above integrated algorithm is verified through static test. In the test, the gyroscope and the accelerometer's short-period precision are about $0.06 \% \mathrm{~h}$ and $10-4 \mathrm{gn}$, GPS receiver can output location, velocity, heading angle, pseudo range, pseudo range ratio and calendar of satellite. For the convenience of analysis, the original data of navigation device is recorder in the test. Calculate the different integrated algorithm about navigation with hardware-in-the-loop method. After dealing with the received GPS information, 0 5S denotes 6 visual satellites, 5S 10S denotes 3 visual satellites, 10S $15 \mathrm{~S}$ recovers to 6 visual satellite again. East velocity error, latitude error and heading error's simulation test is shown as Fig.4-Fig.6.
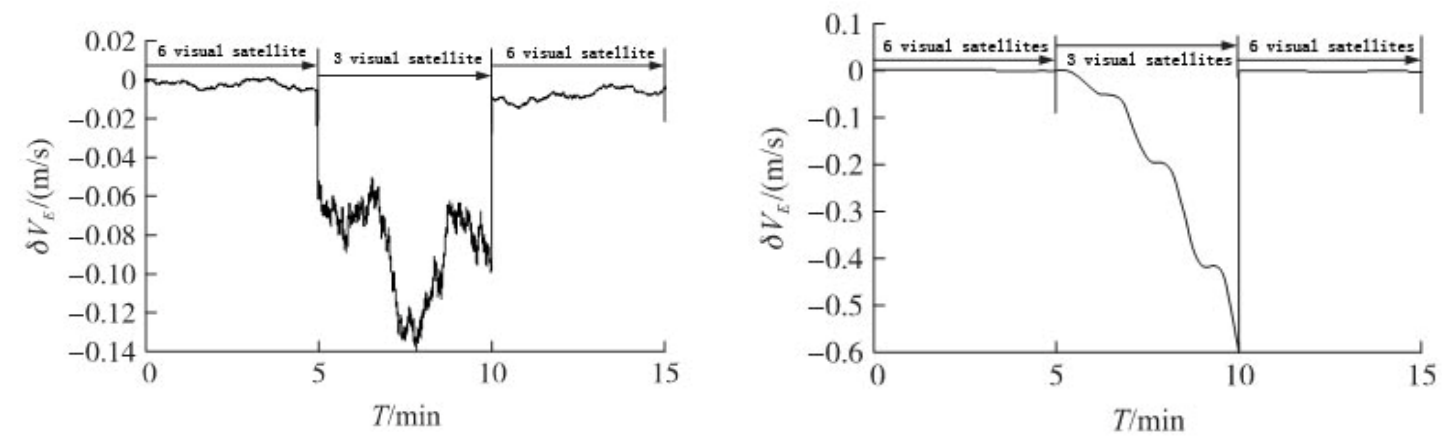

Fig.4 the compare of east velocity error when visual satellites change
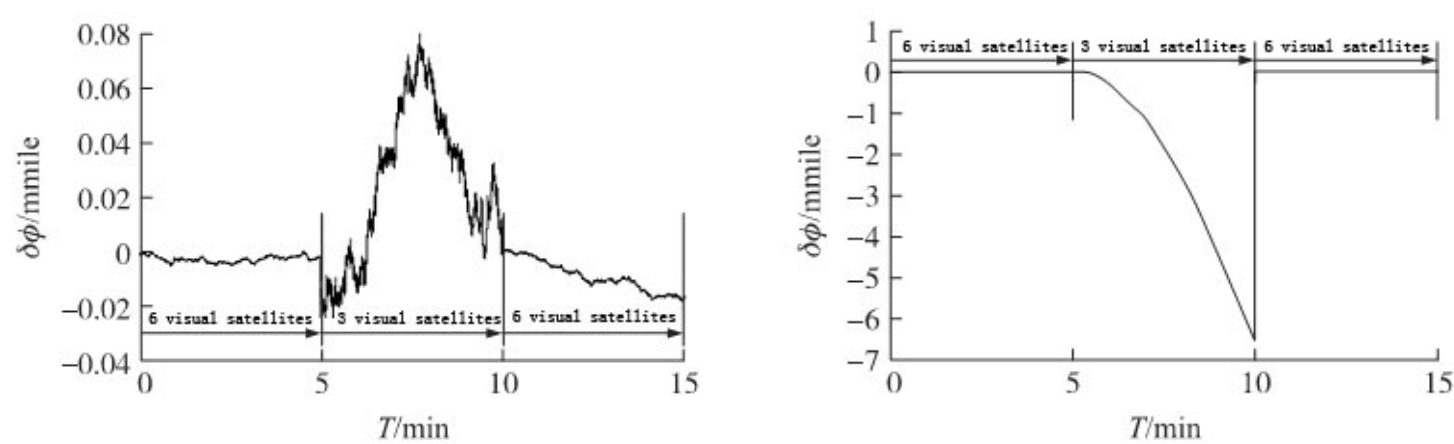

Fig.5 the compare of east latitude error when visual satellites change
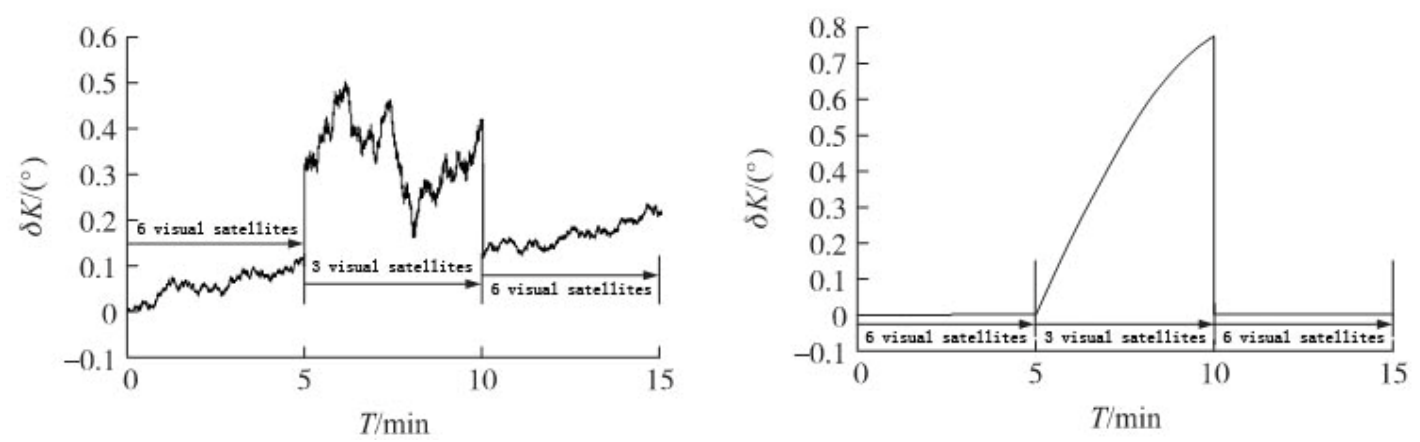

Fig. 6 the compare of heading error when visual satellites change 
From above figure: in the tight system, when the visual satellite number changes to 3 suddenly, the visibility will decrease. Integrated filter output will output one step response. At the same time, the filter procedure doesn't have the obvious scattering trend. So when the visual satellite's number decrease suddenly, pseudo range difference will have one jump change, the pseudo range ration can't be adopted as the system's measurement value. In the program the judgment of pseudo sudden change should be added. When the jump change of pseudo range is detected, it is only the pseudo measurement information that will adjust the system. In the loose integrated system, when the satellites is less than 4, because the loose system has too much dependent upon the GPS, its integrated form will invalid. The system's precision and reliability will rapidly decrease with time goes on. The output of navigation system will scatter.

\section{Reference:}

[1] Zhou Kun-fang, Wu Xi, Kong Jian. Tight integrated GPS/INS character and key technology[J]. China inertial technology transaction, 2009,17 (1): :42-45.

[2] Gong Zhen-chun, Li Pin, Song Zhi-huan. Micro-miniature UAV GPS/MIMU integrated navigation and location research[J]. Aeronautic electric technology, 2004,35 (9):15-34..

[3] Li Peng, Lu Ming-quan, Shen Jun. Integrated navigation system performance simulation and analysis [C].CSNC2010,BeiJing,2010.

[4] Xu Shen-shuo, Shen Peng. SINS/GPS/EMC integrated navigation system information fusion technology research [J].Space navigation technology,2009,29 (2) :41-44. 